\title{
Evaluación de higiene oral y características periodontales en pacientes con obesidad: estudio de casos y controles.
}

\author{
Evaluation of oral hygiene and periodontal characteristics \\ in patients with obesity: case-control study. \\ Dalia Abril Guzmán-Gastelum,* Anabel Huez-González,* \\ Luis Felipe Fornelli-Martín del Campo, ${ }^{\ddagger}$ Sixta Cobos-Floriano, ${ }^{\S}$ Alejandro Donohue-Cornejo," \\ Alma Graciela García-Calderón,* León Francisco Espinosa-Cristóballl
}

\section{RESUMEN}

Introducción: La obesidad sigue siendo considerada un serio problema de salud pública en el mundo. Aunque es bien sabido que esta condición puede aumentar el riesgo para desarrollar o agravar otras condiciones sistémicas; hoy en día, sigue siendo escasa la información que determine los niveles de asociación entre esta enfermedad sistémica con enfermedades orales como la periodontitis. Objetivo: El objetivo de este estudio fue medir la asociación de pacientes obesos con el desarrollo de periodontitis, así como hábitos de higiene oral. Material y métodos: Se realizó un estudio de casos y controles en 24 pacientes obesos y 24 pacientes sin obesidad. Las variables evaluadas fueron hábitos de higiene bucal, profundidad al sondeo, nivel de inserción clínica y nivel de inflamación gingival. Resultados: Los pacientes obesos tuvieron mayor riesgo para desarrollar bajas frecuencias de cepillado bucal diario (70.8\%) y un incremento en la presencia de enfermedad periodontal (70.8\%) comparado con los sujetos no obesos (25.0 y 58.3\%, respectivamente), lo cual determinó asociaciones estadísticamente significativas (baja frecuencia de cepillado $\mathrm{RM}=7.2$, IC $95 \%=2.0-26.1)$ y presencia de enfermedad periodontal $(\mathrm{RM}=1.7, \mathrm{IC}$ $95 \%=0.5-5.7)$. Conclusiones: Es posible que la obesidad actúe como un factor que facilite la aparición de periodontitis por deficiencias en el cepillado dental diario.

Palabras clave: Obesidad, enfermedades periodontales, periodontitis, higiene oral.

\section{ABSTRACT}

Introduction: Obesity is still considered a serious public health problem in the world. Although it is well known that this condition may increase the risk of developing or aggravating other systemic conditions; nowadays, the information that determines the levels of association between this systemic disease with oral diseases such as periodontitis still scarce. Objective: The objective of this study was to measure the association of obese patients with the development of periodontitis as well as oral hygiene habits. Material and methods: A case-control study was conducted in 24 obese patients and 24 patients without obesity. The variables evaluated were oral hygiene habits, depth of probing, clinical attachment level and gingival inflammation. Results: Obese patients had a higher risk of developing low frequencies of daily oral brushing (70.8\%) and an increase in the presence of periodontal disease (70.8\%) compared to non-obese subjects (25.0 and 58.3\%, respectively) determining statistically significant associations (low frequency of brushing $R M=7.2,95 \% C I=2.0-26.1$ ) and presence of periodontal disease $(R M=1.7,95 \% C I=0.5-5.7)$. Conclusions: $I t$ is possible that obesity acts as a factor that facilitates the presence of periodontitis due to deficiencies in daily toothbrushing.

Keywords: Obesity, periodontal diseases, periodontitis, oral hygiene.

\footnotetext{
* Especialidad de Periodoncia, Departamento de Estomatología, Instituto de Ciencias Biomédicas, Universidad Autónoma de Ciudad Juárez. Ciudad Juárez, Chihuahua, México.

‡ Especialidad de Ortodoncia, Departamento de Estomatología, Instituto de Ciencias Biomédicas, Universidad Autónoma de Ciudad Juárez. Ciudad Juárez, Chihuahua, México.

§ Departamento de Contaduría, Instituto de Ciencias Sociales y Administrativas, Universidad Autónoma de Ciudad Juárez. Ciudad Juárez, Chihuahua, México.

" Maestría en Ciencias Odontológicas, Departamento de Estomatología, Instituto de Ciencias Biomédicas, Universidad Autónoma de Ciudad Juárez. Ciudad Juárez, Chihuahua, México.
}

Recibido: 04 Octubre 2019. Aceptado para publicación: 18 Noviembre 2019. 


\section{INTRODUCCIÓN}

L a obesidad es definida como la acumulación de tejido adiposo anormal o excesivo que podría impedir la salud. ${ }^{1}$ En la actualidad, la obesidad se considera como una enfermedad crónica multifactorial que es altamente asociada con múltiples comorbilidades. ${ }^{2}$ Este exceso de peso corporal es considerado como un problema de salud pública a nivel mundial asociado a su gran escala, aceleración en su incremento epidemiológico y a los grandes efectos negativos que impactan la salud como el riesgo de padecer enfermedades crónicas no transmitibles, mortalidad prematura, entre otras. ${ }^{3}$ El indicador usado con mayor frecuencia para la determinación del riesgo en los niveles correctos de tejido adiposo en humanos, de acuerdo con la Organización Mundial de la Salud (OMS), es el índice de masa corporal (IMC), debido a que presenta parámetros fáciles y económicos de usar en estudios clínicos y epidemiológicos. La OMS define numéricamente el sobrepeso como IMC $\geq 25 \mathrm{~kg} / \mathrm{m}^{2}$ y a la obesidad en $\geq 30 \mathrm{~kg} / \mathrm{m}^{2}{ }^{1}$ Se estima que en el año 2030 , $38 \%$ de la población adulta en el mundo podría tener sobrepeso y cerca de $20 \%$ sean sujetos obesos. ${ }^{2}$

En 2015 se reportó que México ocupa el segundo lugar de prevalencia en población adulta a nivel mundial en obesidad, cifras que representan 10 veces más que en países como Japón y Corea; mientras que en la población infantil, nuestro país ocupa el cuarto lugar de prevalencia mundial en obesidad. ${ }^{3}$ En 2016 la Encuesta Nacional de Salud y Nutrición de Medio Camino (ENSANUT MC, desarrollada en México) indicó que 72.5\% de los adultos padecen sobrepeso y obesidad; además, se observó que la prevalencia de obesidad (IMC $\left.\geq 30 \mathrm{~kg} / \mathrm{m}^{2}\right)$ es más alta en el sexo femenino (38.6\%) que en el masculino $(27.7 \%)$, llegando a una obesidad mórbida (IMC $\geq 40.0 \mathrm{~kg} / \mathrm{m}^{2}$ ) en el mismo sexo 2.4 veces más que en los hombres. ${ }^{4}$ En esta misma encuesta, la región norte de México (que incluye los estados de Baja California, Baja California Sur, Coahuila, Chihuahua, Durango, Nuevo León, Sonora, Sinaloa, San Luis Potosí, Tamaulipas y Zacatecas) mostró la mayor prevalencia de obesidad (22.2\%) en comparación con las otras regiones como la región centro, sur, entre otras (11.4-8.2\%). ${ }^{4}$ De acuerdo con información del Instituto de Seguridad y Servicios Sociales de los Trabajadores del Estado (ISSSTE), en 2018, el estado de Chihuahua ha mostrado que $60 \%$ de la población registra un peso corporal por encima de lo normal, mientras que $11.4 \%$ de los niños y $31 \%$ de los adolescentes padecen sobrepeso y algún nivel de obesidad. Asimismo, 6 de cada 10 habitantes en el estado cuentan con sobrepeso y cada uno de ellos presenta uno de los cuatro niveles de obesidad hasta llegar a la obesidad mórbida; de esta manera, Chihuahua se posiciona como uno de los estados con mayores niveles de obesidad en el país. ${ }^{5}$

Por otro lado, las enfermedades periodontales, principalmente la periodontitis, son consideradas una de las principales enfermedades orales asociadas con la presencia de obesidad. ${ }^{6,7}$ La periodontitis es una enfermedad inflamatoria crónica multifactorial de etiología microbiana que afecta a los tejidos que soportan y rodean las estructuras de los dientes. ${ }^{8}$ Factores como la pobre higiene, la acumulación de placa bacteriana (biofilm microbiano), entre otras condiciones podrían favorecer la aparición de periodontitis ${ }^{9}$ sin embargo, otros factores como la variabilidad de la respuesta del huésped a los periodontopatógenos también podrían predisponer la aparición de enfermedades periodontales como la periodontitis. ${ }^{10,11}$ Diversos estudios epidemiológicos han reportado que la obesidad se ha asociado significativamente con la presencia de periodontitis. ${ }^{6,7}$ Características como el IMC, circunferencia de cintura (CC), porcentaje de tejido adiposo subcutáneo y niveles de lípidos en suero han sido consistentemente asociados con el incremento del riesgo de periodontitis comparado con aquellos sujetos con peso normal. ${ }^{12-14}$

Aunque los mecanismos de asociación entre la obesidad y enfermedades periodontales (principalmente la periodontitis) no es completamente claro, estudios han sugerido que la obesidad tiene efectos biológicos dañinos que podrían estar relacionados con la patogénesis de la periodontitis, básicamente por la presencia de mediadores proinflamatorios como el factor de necrosis tumoral alfa (TNF- $\alpha$ ) e interleucinas-6 (IL-6), las cuales pertenecen a la patofisiología de ambas enfermedades. ${ }^{15}$ Estudios en diversas partes del mundo han reportado consistentemente que la obesidad está asociada con el incremento en el riesgo de periodontitis. 6,16,17 En algunos estados del norte de México, como Durango, se ha reportado que los pacientes con algún tipo de diabetes padecían entre $21-48 \%$ de obesidad, mientras que sujetos sin diabetes padecían sólo $12.3 \%$ de obesidad; además, este mismo estudio indicó que la presencia de diabetes mellitus tipo 2 con presencia de obesidad aumentaba significativamente el riesgo de padecer periodontitis. ${ }^{18}$

Sin duda, la periodontitis es una de las manifestaciones más frecuentes en sujetos que padecen obesidad, y esta última se relaciona como un factor de riesgo que puede predisponer la aparición de enfermedades periodontales. A pesar de los diversos estudios relacionados con la presencia de obesidad y periodontitis principalmente, 
existen pocos reportes en la literatura científica de la región del norte de México, particularmente en Ciudad Juárez, Chihuahua, que hayan determinado los niveles de asociación entre la presencia de obesidad y características de higiene oral, así como periodontales que actúen como predisponentes para la presencia de periodontitis. El objetivo de este trabajo fue determinar los niveles de asociación entre la obesidad con parámetros de higiene oral y características clínicas periodontales en sujetos de Ciudad Juárez, Chihuahua, México.

\section{MATERIAL Y MÉTODOS}

Se realizó un estudio de casos y controles en pacientes que asistieron voluntariamente a la Clínica de Admisión del Departamento de Estomatología en el Instituto de Ciencias Biomédicas de la Universidad Autónoma de Ciudad Juárez (UACJ) en Ciudad Juárez, Chihuahua, México, entre abril y octubre de 2017. El estudio fue probado por el Comité de Investigación de la Especialidad de Periodoncia y de la Maestría en Ciencias Odontológicas, ambos pertenecientes a la UACJ. Los sujetos incluidos en el grupo de obesidad fueron con base en los criterios de la OMS, ${ }^{19,20}$ los cuales fueron considerados como pacientes con obesidad cuando el IMC fueran iguales o superiores a $30 \mathrm{~kg} / \mathrm{m}^{2}$, mientras que el grupo con sujetos sin obesidad fue determinado cuando los valores de IMC fueran iguales o menores a $29.9 \mathrm{~kg} / \mathrm{m}^{2}$. Los individuos incluidos para ambos grupos de estudio fueron sujetos varones mayores de 18 años de edad. Los grupos de estudio se generaron de acuerdo con la presencia de la enfermedad: 1) sujetos con obesidad y 2) sujetos sin obesidad (controles). Se excluyeron a sujetos con tratamiento periodontal en los últimos dos meses antes del estudio, con tratamiento ortodóncico previo, fumadores activos, con diagnóstico de diabetes mellitus tipo 1 o 2, hipertensión arterial, alguna otra enfermedad sistémica aparente o haber tomado algún tipo de medicamento dos meses antes del estudio. Todos los sujetos aceptaron voluntariamente su consentimiento informado por escrito. Una historia clínica odontológica y periodontal detallada fueron aplicadas a ciegas evaluando diversas variables. El peso corporal fue medido con una balanza médica y la unidad de medida fue expresada en kilogramos $(\mathrm{kg})$ y la talla o altura de los pacientes fue determinada con un estadímetro incluido en la misma balanza expresado en metros (m). De esta manera, el IMC se calculó dividiendo los kilogramos de peso por el cuadrado de la estatura en metros $\left(\mathrm{IMC}=\right.$ peso $[\mathrm{kg}] /$ estatura $\left.\left[\mathrm{m}^{2}\right]\right){ }^{20}$ El diagnóstico de la enfermedad periodontal se estableció con base en los criterios previamente reportados. ${ }^{21}$ La gingivitis fue diagnosticada cuando existió sólo inflamación de la encía en al menos dos dientes sin presentar evidencia de pérdida de inserción clínica o sin profundidad al sondeo (PS) mayor a $3 \mathrm{~mm}$; la ausencia de estas características determinaron estado de salud periodontal.

La evaluación gingival fue realizada de acuerdo al índice gingival (IG) ${ }^{22}$ conforme a los siguientes parámetros: 0 = encía sana; 1 = inflamación leve, cambio en la coloración, ligeramente edematizada y sin sangrado al sondeo; 2 = inflamación moderada, encía roja brillante, congestionada sin puntilleo y hemorragia a los 30 segundos posteriores al sondeo; 3 = inflamación severa, edema, congestión, hemorragia espontánea, zonas ulceradas y rojo intenso. La evaluación para la acumulación de biofilm (placa bacteriana) y cálculo dental se determinó a través del índice de higiene oral simplificado (IHOS), ${ }^{23}$ en el cual los valores para placa $(0=$ ausencia de placa; $1=$ residuos blandos que cubren menos de un tercio de la superficie del diente; 2 = residuos blandos y cálculo dental que cubren más de la tercera parte, pero menos de dos terceras partes de la superficie dental; 3 = residuos que cubren más de las dos terceras partes) y cálculo dental $(0=$ ausencia de cálculo subgingival; 1 = presencia de cálculos subgingival cubriendo más de un tercio de la superficie examinada; 2 $=$ presencia de cálculo subgingival cubriendo más de un tercio, pero menos de dos tercios; 3 = presenta cálculo en subgingival más de dos tercios de la superficie examinada) fueron obtenidos sumando los valores y divididos entre el número de superficies examinadas. La evaluación periodontal fue registrada en un periodontograma, el cual incluyó la profundidad al sondeo (PS), nivel de inserción clínica, ambos expresados en milímetros, así como la presencia de sangrado durante el sondeo (tipo de sonda periodontal y calibración clínica de los operadores).

\section{Análisis estadístico}

Los datos fueron evaluados en frecuencias, porcentajes, promedios y desviación estándar. El análisis entre grupos se realizó con el análisis de $\chi^{2}$ y las diferencias entre grupos fueron determinadas cuando $p<0.05$. Las asociaciones de riesgo se determinaron con el análisis de razón de momios, con un intervalo de confianza del 95\%. El programa estadístico utilizado fue IBM-SPSS Statistics versión 25.

\section{RESULTADOS}

Los pacientes evaluados en el estudio fueron un total de 48 sujetos (24 sujetos obesos y 24 sujetos no obesos) 
prevaleciendo en general el género masculino (100\%). La edad promedio de los pacientes fue de $38.7 \pm 14.5$ años de edad. El grupo de pacientes obesos fue ligeramente menor en la edad promedio con $36.25 \pm 10.2$ años que aquellos sujetos no obesos con $41.3 \pm 17.8$ años; sin embargo, ninguna diferencia estadística fue determinada $(p>0.05)$.

La Tabla 1 muestra los resultados de la frecuencia del cepillado diario y la distribución del nivel de acúmulo de biofilm (placa) y cálculo dental por el IHOS. Se puede observar que los sujetos obesos tuvieron bajas frecuencias de cepillado diario en comparación con los sujetos no obesos; mientras que aquéllos sin obesidad demostraron cepillarse los dientes más veces al día que los pacientes con obesidad. Además, diferencias significativas fueron encontradas en la frecuencia del cepillado y los grupos de estudio ( $p<0.05$ ). Por otro lado, el nivel bajo y muy bajo de acumulación de biofilm microbiano y cálculo dental fue ligeramente menor en pacientes obesos que en los sujetos no obesos, mientras que la acumulación alta y muy alta fue observada similar en ambos sujetos. Al realizar la comparación independiente entre los grupos, ninguna diferencia significativa fue encontrada ( $p>0.05$ ). Lo anterior indica que los pacientes obesos y los no obesos tienen estadísticamente el mismo nivel de acumulación de placa bacteriana y cálculo dental; sin embargo, los sujetos obesos tienden a desarrollar significativamente bajas frecuencias de cepillado dental comparado a los sujetos no obesos.

En la Tabla 2 se muestra la distribución de las características periodontales de los sujetos obesos y no obesos. La distribución de la presencia de las diversas enfermedades periodontales determinó que los sujetos sin obesidad fueron periodontalmente más sanos que los pacientes con obesidad, mientras que los sujetos obesos tuvieron mayores frecuencias de gingivitis y periodontitis que aquéllos no obesos. Por otro lado, observamos que en la profundidad al sondeo en los pacientes obesos se obtuvieron promedios mayores que los sujetos no obesos. En contraste, el nivel de inserción clínica para los sujetos obesos fue ligeramente menor que los pacientes no obesos. Aunque la distribución de la presencia y severidad de la enfermedad periodontal, así como los promedios de la profundidad al sondeo y el nivel de inserción clínica variaron entre los sujetos obesos y no obesos, el comportamiento de todas estas variables fue estadísticamente similar entre los pacientes obesos y no obesos ( $p>0.05)$.

En la Tabla 3 se observan los resultados de las asociaciones de riesgo encontradas entre los pacientes obesos y no obsesos relacionadas con la frecuencia del cepillado, acumulación de biofilm de placa bacteriana y cálculo dental, así como de la presencia de enfermedad periodontal. El grupo de sujetos obsesos mostró mayores deficiencias en el cepillado diario comparado con los sujetos no obesos. Además, la presencia de enfermedad periodontal fue presentada con mayor frecuencia en los pacientes obesos que en los no obesos. Las asociaciones de riesgo significativas entre los sujetos obesos y no obesos fueron encontradas entre la baja frecuencia del cepillado y la presencia de enfermedad periodontal, pero ninguna asociación de riesgo significativa fue determinada para la

Tabla 1: Distribución de la frecuencia del cepillado bucal y del IHOS en los grupos de estudio $(n=48)$.

Obesos $(n=24)$

$\mathrm{n}(\%)$
No obesos $(\mathrm{n}=24)$

$\mathrm{n}(\%)$

$0(0.0)$

$6(25.0)$

$14(58.3)$

$4(16.7)$

$11(45.8)$

$9(37.5)$

4 (16.7)

$0(0.0)$ p

0.003

7 (29.2)

$13(54.2)$

$7(29.2)$

4 (16.7)

$0(0.0)$
.

IHOS = índice de higiene oral simplificado. 
Tabla 2: Características periodontales de los sujetos obesos y no obesos $(n=48)$.

\begin{tabular}{|c|c|c|c|}
\hline & $\begin{array}{c}\text { Obesos }(n=24) \\
n(\%)\end{array}$ & $\begin{array}{c}\text { No obesos }(n=24) \\
n(\%)\end{array}$ & $\mathrm{p}$ \\
\hline \multicolumn{4}{|l|}{ Diagnóstico } \\
\hline Sano & 7 (29.2) & $10(41.7)$ & \\
\hline Gingivitis & $10(41.7)$ & $9(37.5)$ & 0.633 \\
\hline Periodontitis & 7 (29.2) & $5(20.8)$ & \\
\hline PS (mm) & $1.45 \pm 0.65$ & $1.08 \pm 0.82$ & 0.110 \\
\hline NIC (mm) & $3.27 \pm 1.05$ & $3.40 \pm 1.50$ & 0845 \\
\hline
\end{tabular}

PS = profundidad al sondeo; NIC = nivel de inserción clínica.

Tabla 3: Asociaciones de riesgo entre los sujetos obesos y no obesos $(n=48)$.

\begin{tabular}{|c|c|c|c|}
\hline & $\begin{array}{c}\text { Obesos }(n=24) \\
n(\%)\end{array}$ & $\begin{array}{c}\text { No obesos }(n=24) \\
n(\%)\end{array}$ & RM (IC 95\%) \\
\hline Baja frecuencia de cepillado (0-2 veces por día) & $17(70.8)$ & $6(25.0)$ & $7.2(2.0-26.1)$ \\
\hline Alto acúmulo de placa microbiana y cálculo (IHOS 2-3) & $4(16.7)$ & $4(16.7)$ & $1.0(0.2-4.5)$ \\
\hline Enfermedad periodontal (gingivitis o periodontitis) & $17(70.8)$ & $14(58.3)$ & $1.7(0.5-5.7)$ \\
\hline Enfermedad periodontal activa (PS > $3 \mathrm{~mm}$ ) & $0(0.0)$ & $0(0.0)$ & - \\
\hline Alta experiencia de enfermedad periodontal (NIC $>3 \mathrm{~mm}$ ) & $14(58.3)$ & $14(58.3)$ & $1.0(0.31-3.1)$ \\
\hline
\end{tabular}

$\mathrm{RM}$ = razón de momios; IC = intervalo de confianza del 95\%; IHOS = índice de higiene oral simplificado; PS = profundidad al sondeo; NIC = nivel de inserción clínica.

alta acumulación de placa y cálculo dental, enfermedad periodontal activa ni para la alta experiencia de enfermedad periodontal. Lo anterior indica que el ser obeso podría incrementar, al menos, siete veces más el riesgo de tener bajas frecuencias del cepillado diario, así como de aumentar casi dos veces más el riesgo de desarrollar alguna enfermedad periodontal comparado con aquellos pacientes que no presentan obesidad; sin embargo, la presencia activa y la alta experiencia de la enfermedad periodontal fueron independientes de la presencia de la obesidad.

\section{DISCUSIÓN}

El presente estudio determinó asociaciones de riesgo significativas entre la baja frecuencia del cepillado diario y la presencia de enfermedad periodontal en sujetos con presencia de obesidad; empero, la obesidad no se relacionó con el nivel de profundidad al sondeo, el nivel de inserción clínica, ni con la alta acumulación de placa bacteriana y cálculo dental. Esto sugiere que la presencia de obesidad puede incrementar el desarrollo de malos hábitos de higiene oral a través de la disminución significativa de la frecuencia del cepillado diario y de esta manera la aparición de enfermedades periodontales como gingivitis y periodontitis, pero no se podría relacionar con el desarrollo de alteraciones periodontales como la profundidad al sondeo y el nivel de inserción clínica. Estos datos deben ser difundidos a la población en general y a los prestadores de salud, dado que en México existe un problema relacionado con la obesidad, según lo publicado en la Encuesta Nacional de Salud del año 2016. ${ }^{24}$

Un estudio realizado en 80 sujetos obesos, 69 con sobrepeso y 89 sujetos con peso normal determinó que la periodontitis severa fue significativamente asociada con los sujetos obesos $(\mathrm{RM}=3.25,95 \% \mathrm{Cl}=1.27-8.31, \mathrm{p}=$ 
0.01), pero no se asoció con los pacientes con sobrepeso $(p=0.59)$, concluyendo que los pacientes con obesidad deberían ser referidos a una evaluación clínica periodontal. ${ }^{25}$ Otro estudio determinó la relación entre el índice de masa corporal y la periodontitis en mujeres postmenopausia, encontrando que las pacientes con obesidad con un índice de masa corporal mayor a 25 disminuyeron el riesgo para tener periodontitis comparado con personas de peso normal $(\mathrm{RM}=0.54$; IC 95\% $=0.27-0.87)$; además, reportaron que los sujetos obesos mostraron una mayor pérdida de la inserción clínica, mayor acúmulo de cálculo y biofilm microbiano comparado con los sujetos sin obesidad $(p<0.01){ }^{26}$

Asimismo, se ha evaluado la asociación entre el síndrome metabólico y la enfermedad periodontal en 657 mujeres postmenopáusicas entre los 50 y 79 años de edad, indicando que el síndrome metabólico se asoció estadísticamente con la acumulación de biofilm acumulado supragingivalmente $(\mathrm{RM}=1.74$; IC $95 \%=1.22-2.50)$; $\sin$ embargo, no se asoció significativamente con la presencia de periodontitis de acuerdo con la profundidad al sondeo y el nivel de inserción clínica ( $p>0.05$ ), concluyendo que la relación entre la presencia del síndrome metabólico y la periodontitis no fue determinada. ${ }^{27}$ Un estudio menos reciente publicado en 2009 evaluó 878 mujeres y 719 hombres entre los 20 y 95 años de edad en los cuales los sujetos obesos tuvieron menores asociaciones de riesgo para el nivel de inserción clínica que aquellos pacientes con peso corporal normal (RM $=0.60$; IC 95\% $=0.36-0.99$ ), mientras que los sujetos con sobrepeso tuvieron mayores asociaciones de riesgo para presentar sangrado durante el sondeo que los sujetos con peso normal $(\mathrm{RM}=1.36$; IC 95\% = 1.04-1.78); este estudio concluyó que un específico índice de masa corporal de los sujetos podría estar inversamente asociada con el nivel de inserción y con la presencia de sangrado durante el sondeo. ${ }^{28}$ Adicionalmente, otro estudio reportó que los pacientes diabéticos presentaron mayor frecuencia de obesidad (23.1-41.9\%) que los sujetos sin diabetes (14\%), se mostró que los pacientes diabéticos tuvieron mayores asociaciones de riesgo para desarrollar periodontitis (RM $=2.85, \mathrm{IC} 95 \%=0.18-6.8, \mathrm{p}<0.05)$ que los sujetos $\sin$ diabetes. Además, los pacientes con diabetes mellitus tipo 2 con obesidad mostraron tener mayores asociaciones de riesgo que pacientes con diabetes mellitus tipo $1 \mathrm{con}$ obesidad ( $R M=7.4$, IC 95\% = 1.93-28.49, $\mathrm{p}<0.05$ ).

Nuestros resultados indicaron que los sujetos obesos mostraron bajas frecuencias del cepillado dental realizado por día (70.8\%) y la presencia incrementada de enfermedades periodontales (70.8\%) que los sujetos sin obesidad
(25 y $28.3 \%$, respetivamente) determinando asociaciones de riesgo significativas entre la baja frecuencia del cepiIlado ( $\mathrm{RM}=7.2$, IC 95\% = 2.0-26.1) y la presencia de enfermedad periodontal $(R M=1.7, \mathrm{IC} 95 \%=0.5-5.7)$ en los sujetos obesos; sin embargo, la variación de las alteraciones periodontales como la profundidad al sondeo y el nivel de inserción clínica, así como la alta acumulación de biofilm microbiano y cálculo dental demostraron no tener alguna asociación estadísticamente significativa entre los grupos $(R M=1.0, \mathrm{IC} 95 \%=0.2-4.5, \mathrm{p}>0.05)$. Esto puede sugerir que los pacientes con obesidad podrían tener más riesgo de desarrollar deficiencias del cuidado bucal, principalmente ocasionado por la disminución de la frecuencia del hábito del cepillado y, además, el riesgo incrementado para el desarrollo de las distintas enfermedades periodontales, independientemente de la severidad actual de la enfermedad y la presencia previa a alguna enfermedad periodontal, principalmente periodontitis. Es posible que la presencia de obesidad en estos sujetos relacionada con la baja frecuencia del cepillado y a la presencia de enfermedades periodontales se deba a factores psicosociales como la depresión, la cual se asocia consistentemente con la ganancia gradual del peso corporal, la aparición misma de la obesidad, empeoramiento del sistema endocrino como la resistencia a la insulina, así como la aparición de otras enfermedades sistémicas como la diabetes mellitus tipo 2. ${ }^{29-32}$ Además, la depresión podría afectar complementariamente el riesgo de desarrollar diabetes mellitus tipo 2 derivado del impacto en la adiposidad; de esta forma, la depresión en pacientes obesos podría desarrollar un efecto en la resistencia de la insulina a través del comportamiento relacionado con el estrés, así como otros mecanismos psicosociales como lo son la alimentación emocional excesiva, inactividad física, alteración del sueño, desregulación del eje adrenopituitario-hipotalámico y/o con presencia de inflamación crónica; ${ }^{31}$ además, estos factores podrían generar al mismo tiempo la aparición de enfermedades periodontales como gingivitis y periodontitis. ${ }^{33}$

Es bien sabido que la periodontitis tiene una gran influencia sobre la patogénesis de ciertas enfermedades sistémicas que puede incluir la diabetes mellitus, principalmente el tipo 2 , y la obesidad. ${ }^{27,33}$ La periodontitis es una enfermedad crónica inflamatoria de los tejidos periodontales estimulada por microorganismos patógenos subgingivales principalmente. Esta respuesta inflamatoria es caracterizada por una secreción no regulada de mediadores inflamatorios y de destrucción tisular pertenecientes al huésped. Los más estudiados son la interleucina (IL) 1b, IL-6, prostaglandina E2 (PGE2), factor de necrosis tumoral 
alfa (TNF- $\alpha$ ), RANKL y las metaloproteinasas de matriz extracelular (MMP), particularmente MMP-8, MMP-9 y MMP-13, así como células $T$ reguladoras de citocinas (por ejemplo, IL-12, IL-18) y de quimiocinas. ${ }^{34}$ Por otro lado, se sabe que los mecanismos biológicos relevantes entre la obesidad y la periodontitis involucra citocinas derivadas del tejido adiposo y hormonas conocidas como adipocinas. ${ }^{35}$ Generalmente, la obesidad es asociada con estados inflamatorios crónicos de bajo grado, debido al incremento de la expresión de adipocinas proinflamatorias (TNF- $\alpha$, IL-6, leptina, resistina, otras) y una expresión disminuida de adipocinas antiinflamatorias; además de las numerosas adipocinas con efecto proinflamatorio, el tejido adiposo secreta un número reducido de factores antiinflamatorios incluyendo la adiponectina. ${ }^{36}$ Diversos investigadores coinciden que los mediadores más importantes asociados entre la obesidad y la periodontitis son el TNF- $\alpha$ y la IL-6, los cuales están involucrados en la patofisiología de ambas enfermedades, principalmente causando la destrucción de los tejidos periodontales. ${ }^{15,35}$ A pesar de toda la información disponible sobre la relación entre la obesidad y la enfermedad periodontal, con este estudio se puede sugerir que esta relación es posiblemente debido a la deficiencia en el cepillado dental y esto aumenta el número de bacterias asociadas con la enfermedad, principalmente periodontitis, facilitando la secreción de mediadores proinflamatorios como el TNF- $\alpha$ y la IL-6, destruyendo de esta manera los tejidos periodontales y finalmente concluya con la aparición de las enfermedades periodontales como la periodontitis.

Adicionalmente, la relación entre la obesidad y la periodontitis sigue siendo investigada, por estar razón es necesario la utilización de nuevas y diferentes técnicas biológicas y la inclusión de otros factores que pueden intervenir en el desarrollo de las enfermedades periodontales $y$, al mismo tiempo, la asociación de otras enfermedades sistémicas altamente relacionadas con la obesidad como la diabetes mellitus tipo 2 .

Este estudio demostró asociaciones significativas entre obesidad, frecuencia de cepillado bucal diario disminuida y la presencia de enfermedades periodontales; sin embargo, marcadores como la profundidad al sondeo y el nivel de inserción clínica se mostraron similares en sujetos con y sin obesidad.

\section{CONCLUSIONES}

La presencia de la obesidad facilita el desarrollo de periodontitis, principalmente por disminución de la frecuencia del cepillado bucal constante y no precisa- mente por la severidad de la enfermedad ni por la experiencia de enfermedad periodontal. Estos resultados deberían ser difundidos en la población en general y a los prestadores de servicios médico-odontológico para la prevención, control y tratamiento de las enfermedades periodontales como la periodontitis en pacientes con obesidad.

\section{AGRADECIMIENTOS}

Los autores agradecen al Departamento de Estomatología del Instituto de Ciencias Biomédicas de la Universidad Autónoma de Ciudad Juárez por el apoyo para la realización de este trabajo.

\section{BIBLIOGRAFÍA}

1. WHO. Obesity and other diet-related chronic diseases list of publications. Geneva, Switzerland: WHO; 2015.

2. Smith KB, Smith MS. Obesity Statistics. Prim Care Clin Off Pract. 2016; 43 (1): 121-135. doi: 10.1016/j.pop.2015.10.001

3. Dávila-Torres J, González-Izquierdo JJ, Barrera-Cruz A. Obesity in Mexico. Rev Med Inst Mex Seguro Soc. 2015; 53 (2): 240-249.

4. Encuesta Nacional de Salud y Nutrición de Medio Camino 2016. Secretaría de Salud. [Acceso 2 de octubre de 2019] Disponible en: gob.mx.https://www.gob.mx/salud/documentos/encuesta-nacionalde-salud-y-nutricion-de-medio-camino-2016.

5. Chihuahua primer lugar mundial en obesidad. El Heraldo de Chihuahua. Publicado en 2018. [Acceso 4 de octubre de 2019] Disponible en: https://www.elheraldodechihuahua.com.mx/local/ chihuahua-primer-lugar-mundial-en-obesidad-2850381.html.

6. Chaffee BW, Weston SJ. Association between chronic periodontal disease and obesity: a systematic review and meta-analysis. J Periodontol. 2010; 81 (12): 1708-1724.

7. Guh DP, Zhang W, Bansback N, Amarsi Z, Birmingham CL, Anis AH. The incidence of Co-morbidities related to obesity and overweight: a systematic review and meta-analysis. BMC Public Health. 2009; 9 (1): 88.

8. Babay N, Alshehri F, Al Rowis R. Majors highlights of the new 2017 classification of periodontal and peri-implant diseases and conditions. Saudi Dent J. 2019; 31 (3): 303-305.

9. Van Dyke TE, Sheilesh D. Risk factors for periodontitis. J Int Acad Periodontol. 2005; 7 (1): 3-7.

10. Knight ET, Liu J, Seymour GJ, Faggion CM, Cullinan MP. Risk factors that may modify the innate and adaptive immune responses in periodontal diseases. Periodontol 2000. 2016; 71 (1): 22-51. doi: 10.1111/prd.12110

11. Kornman KS, Page RC, Tonetti MS. The host response to the microbial challenge in periodontitis: assembling the players. Periodontol 2000. 1997; 14: 33-53.

12. Saito T, Shimazaki $Y$, Sakamoto $M$. Obesity and periodontitis. $N$ Engl J Med. 1998; 339 (7): 482-483.

13. Khader YS, Bawadi HA, Haroun TF, Alomari M, Tayyem RF. The association between periodontal disease and obesity among adults in Jordan. J Clin Periodontol. 2009; 36 (1): 18-24.

14. Al-Zahrani MS, Bissada NF, Borawski EA. Obesity and periodontal disease in young, middle-aged, and older adults. J Periodontol. 2003; 74 (5): 610-615. 
15. Genco RJ, Grossi SG, Ho A, Nishimura F, Murayama Y. A proposed model linking inflammation to obesity, diabetes, and periodontal infections. J Periodontol. 2005; 76 (11-s): 2075-2084.

16. Han DH, Lim SY, Sun BC, Paek DM, Kim HD. Visceral fat areadefined obesity and periodontitis among Koreans. J Clin Periodontol. 2010; 37 (2): 172-179.

17. Haffajee AD, Socransky SS. Relation of body mass index, periodontitis and Tannerella forsythia. J Clin Periodontol. 2009; 36 (2): 89-99.

18. Peralta D, Espinosa L, Carreón R et al. Evaluación de la enfermedad periodontal e hiperglucemia asociados con diabetes mellitus tipo 2 en pacientes del estado Durango, México. Rev ADM. 2016; 73 (2): $72-80$.

19. OMS. 10 datos sobre la obesidad. [Acceso 2 de octubre de 2019] Disponible en: https://www.who.int/features/factfiles/obesity/facts/es/.

20. WHO. Obesity. Geneva: WHO; 2014.

21. Armitage GC. Development of a classification system for periodontal diseases and conditions. Ann Periodontol. 1999; 4 (1): 1-6. doi: 10.1902/annals.1999.4.1.1.

22. Löe $H$. The gingival index, the plaque index and the retention index systems. J Periodontol. 1967; 38 (6): 610-616.

23. Greene JG, Vermillion JR. The simplified oral hygiene index. J Am Dent Assoc. 1964; 68 (1): 7-13. doi: 10.14219/jada. archive.1964.0034

24. Encuesta Nacional de Salud y Nutrición. Publicado en 2016. [Acceso 25 de septiembre de 2019] Disponible en: https://ensanut.insp.mx/ ensanut2016/index.php

25. Santos T, Cury PR, Santos E, Vasconcelos R, Santos JN Dos, Ramalho LMP. Association between severe periodontitis and obesity degree: a preliminary study. Oral Health Prev Dent. 2019; 17 (2): 173-177.

26. Habashneh R AL, Azar W, Shaweesh A, Khader Y. The relationship between body mass index and periodontitis among postmenopausal women. Obes Res Clin Pract. 2016; 10 (1): 15-23.

27. LaMonte MJ, Williams AM, Genco RJ et al. Association between metabolic syndrome and periodontal disease measures in postmenopausal women: the buffalo osteoperio study. J Periodontol. 2014; 85 (11): 1489-1501.

28. Kongstad J, Hvidtfeldt UA, Grønbæk M, Stoltze K, Holmstrup P. The relationship between body mass index and periodontitis in the copenhagen city heart study. J Periodontol. 2009; 80 (8): 12461253.

29. Gulley LD, Shomaker LB, Kelly NR et al. Indirect effects of a cognitive-behavioral intervention on adolescent weight and insulin resistance through decreasing depression in a randomized controlled trial. J Pediatr Psychol. 2019; 44 (10): 1163-1173.

30. Hannon TS, Rofey DL, Lee S, Arslanian SA. Depressive symptoms and metabolic markers of risk for type 2 diabetes in obese adolescents. Pediatr Diabetes. 2013; 14 (7): 497-503.

31. Markowitz S, Friedman MA, Arent SM. Understanding the relation between obesity and depression: causal mechanisms and implications for treatment. Clin Psychol Sci Pract. 2008; 15 (1): 1-20.

32. Nemiary D, Shim R, Mattox G, Holden K. The relationship between obesity and depression among adolescents. Psychiatr Ann. 2012; 42 (8): 305.

33. Bascones-Martínez A, Muñoz-Corcuera M, Bascones-llundain J. Diabetes and periodontitis: a bidirectional relationship. Med Clin (Barc). 2015; 145 (1): 31-35.

34. Preshaw PM, Alba AL, Herrera D et al. Periodontitis and diabetes: a two-way relationship. Diabetologia. 2012; 55 (1): 21-31.

35. Akram Z, Abduljabbar T, Abu Hassan MI, Javed F, Vohra F. Cytokine profile in chronic periodontitis patients with and without obesity: a systematic review and meta-analysis. Dis Markers. 2016; 2016: 4801418.

36. Nakamura K, Fuster JJ, Walsh K. Adipokines: a link between obesity and cardiovascular disease. J Cardiol. 2014; 63 (4): 250-259.

\section{Correspondencia:}

Dr. en C. León Francisco Espinosa Cristóbal Envolvente del PRONAF, Zona PRONAF, C.P. 32310, Ciudad Juárez, Chihuahua, México. Tel: (+52) 6566881800

E-mail: leohamet@hotmail.com 